\title{
Performance of an I- SANEX system based on a water-soluble BTP under continuous irradiation in a y- radiolysis test loop
}

D.R. Peterman, A. Geist, B.J. Mincher, G. Modolo, H. Galán, L.G. Olson, R.G. McDowell

September 2016

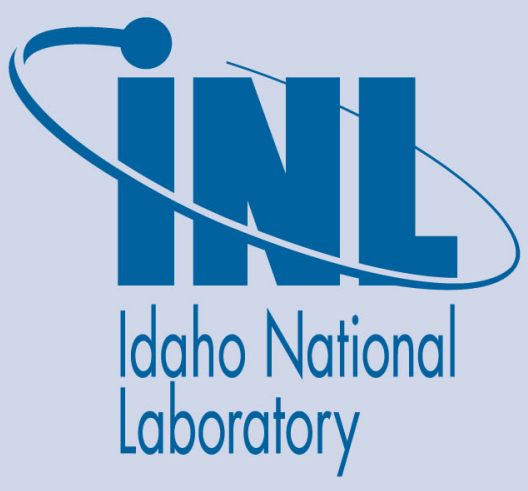

The INL is a U.S. Department of Energy National Laboratory operated by Battelle Energy Alliance 


\section{Performance of an I-SANEX system based on a water- soluble BTP under continuous irradiation in a $y$ - radiolysis test loop}

D.R. Peterman, A. Geist, B.J. Mincher, G. Modolo, H. Galán, L.G. Olson, R.G. McDowell

September 2016

Idaho National Laboratory Idaho Falls, Idaho 83415

http://www.inl.gov

Prepared for the

U.S. Department of Energy

Under DOE Idaho Operations Office

Contract DE-AC07-05ID14517 


\section{Performance of an $i$-SANEX system based on a}

\section{water-soluble BTP under continuous irradiation in a}

\section{$\gamma$-radiolysis test loop}

Dean Peterman, ${ }^{a}$ Andreas Geist,${ }^{b}$ Bruce Mincher, ${ }^{a}$ Giuseppe Modolo ${ }^{c}$, Maria Hitos. Galán, ${ }^{d}$ Lonnie Olson, ${ }^{a}$ Rocklan McDowell ${ }^{a}$

a Idaho National Laboratory, Aqueous Separations and Radiochemistry Department, Idaho Falls, ID, USA 83415

${ }^{\mathrm{b}}$ Karlsruhe Institute of Technology (KIT), Institute for Nuclear Waste Disposal (INE), P. O. Box 3640, 76021 Karlsruhe, Germany

c Forschungszentrum Jülich GmbH (FZJ), Institute of Energy and Climate Research, Nuclear Waste Management and Reactor Safety (IEK-6), 52425 Jülich, Germany

${ }^{\mathrm{d}}$ Centro de Investigaciones Energéticas, Medioambientales y Tecnológicas (CIEMAT), Spain.

\section{ABSTRACT}

The $i$-SANEX solvent formulation consisting of nominally $0.2 \mathrm{M}$ TODGA/5\% 1 octanol/dodecane was g-irradiated under realistic conditions in the INL radiolysis test loop, in contact with both extraction and stripping aqueous phases. The extraction aqueous phase was 4.5 
$\mathrm{M} \mathrm{HNO}_{3}$, and the stripping aqueous phase was $0.018 \mathrm{M} \mathrm{SO}_{3}$-Ph-BTP/0.35 $\mathrm{M} \mathrm{HNO}_{3}$. When irradiated in contact with only the $4.5 \mathrm{M} \mathrm{HNO}_{3}$ phase, the TODGA solvent maintained excellent extraction performance for americium, cerium, europium and neodymium to a maximum absorbed dose of nearly $0.9 \mathrm{MGy}$. The results for preliminary static, batch irradiations were consistent with test loop findings. When the aqueous phase was changed to that containing the aqueous soluble BTP, the irradiated aqueous phase showed a dramatic color change, but this does not appear to have had adverse effect on solvent extraction performance. The distribution ratios for both the lanthanides and actinides were invariant with absorbed dose, and the separation factors were essentially unchanged to a maximum absorbed dose of $174 \mathrm{kGy}$. The results of ICP-MS analysis of the aqueous and organic phases showed that ruthenium and strontium were not extracted in the presence of the irradiated BTP-aqueous phase. Molybdenum was extracted under both conditions. Further, the build-up of corrosion products of stainless steel in the presence of the BTP was not dramatically greater than in the nitric acid-only system. Phase separation times were not adversely affected by irradiation for either system. The results presented here indicate that the performance of the TODGA/SO $\mathrm{SO}_{3}$-Ph-BTP, $i$-SANEX process under test loop radiolysis is much better than expected based on a literature report using singlephase batch irradiation experiments, and better than might be predicted base on DGA radiolysis studies alone.

\section{INTRODUCTION}

The development of a process solvent extraction system capable of partitioning trivalent actinides from trivalent lanthanides is probably the highest aspiration of the fuel cycle separations community. Active research programs are working to solve this problem in Europe, North America and Asia. The common approach uses soft donor compounds, mainly with N- 
donor functional groups, to selectively complex the actinides. A bewildering series of acronyms has evolved as incremental improvements were made on a series of solvent formulations at the various research institutions. Avoiding the acronyms, early multi-cycle approaches extracted the lanthanides and actinides from the fuel dissolution using a non-selective O-donor as a group $f$ element complexing agent dissolved in an aliphatic diluent. Examples of such group extraction ligands include malonamides, diglycolamides and organo-phosphine oxides. The actinides and lanthanides were then back extracted (stripped) to a new, mildly acidic aqueous phase. Then, the actinides were re-extracted into a new organic phase using an $\mathrm{N}$-donor ligand, such as a bistriazinylbipyridine (BTBP) compound. ${ }^{1,2}$

Simplification of such a multi-cycle process to a single cycle saves money and complexity, and this is always important in nuclear applications. In a single cycle, the actinides in the actinide/lanthanide-loaded organic phase are stripped to a buffered aqueous phase, usually using polyaminocarboxylate N-donor reagents. ${ }^{3}$ Subsequent developments included the use of acidic extractants in combination with neutral species in the organic phase, to better hold the lanthanides in that phase even when stripping the actinides with buffered aqueous phases of low acidity. A number of combinations of such organic and aqueous complexing agents have been used; for examples see: Modolo et al., ${ }^{1-2}$ Gannaz et al., ${ }^{4}$ and Gelis and Lumetta. ${ }^{5}$ A summary of these developments was recently presented by Wilden et al. ${ }^{6}$

The actinide-selective N-donor BTBPs and bistriazinylpyridines (BTPs) are typically used in the organic phase for group actinide extraction purposes. ${ }^{1-2,}$ 7,7b However, a very recent development conceived at Karlruhe Institute of Technology (KIT), Germany, is the functionalization of a BTP with sulfonyl groups, to make it soluble in the aqueous phase. The aqueous soluble N-donor is (2,6-bis-(5,6-di(sulfophenyl)-1,2,4-triazin-3-yl)-pyridine, or $\mathrm{SO}_{3}$-Ph- 
BTP, shown in Figure $1 .^{8}$ In combination with an organic phase diglycolamide O-donor actinide/lanthanide group extraction ligand (the well-known tetraoctyldiglycolamide (TODGA) shown in Figure 1. $)^{9,10}$ the separation would then be accomplished in a single cycle referred to as the $i$-SANEX (innovative Selective ActiNide EXtraction) process. This process has been tested at the lab scale at Forschungszentrum Jülich (FZJ), Germany, using centrifugal contactors and it resulted in $99.8 \%$ recovery of $\mathrm{Am}$ and $\mathrm{Cm}$ from a synthetic high active raffinate solution containing lanthanides and other constituents expected to remain in dissolved nuclear fuel following the initial conventional uranium extraction process. ${ }^{6}$

While this result is promising, in addition to adequate solvent extraction performance any solvent system used in the processing of dissolved nuclear fuel must be robust in a high radiation environment. ${ }^{11}$ Unfortunately, the water soluble BTP was recently reported to be sensitive to $\gamma$ radiolysis when the aqueous phase was batch irradiated using a ${ }^{60} \mathrm{Co}$ source. ${ }^{12}$ However, in that preliminary work, only the BTP-containing aqueous phase was irradiated. In the actual biphasic process, multiple competing free radical reactions occur to either degrade or protect the solvent extraction ligands. Similarly, the rapid depletion of dissolved oxygen in irradiated sealed samples may alter the rate of ligand degradation or ligand radiolysis products generated as compared to the aerated process system. ${ }^{13}$ Therefore, in this study the biphasic $i$-SANEX solvent system was irradiated with continuous mixing and recirculation in a test loop based on a Nordion GammaCell ${ }^{60} \mathrm{Co}$ irradiator, constructed at the Idaho National Laboratory, USA. The loop was designed to irradiate mixed phases with recirculation under process-like conditions. This $i$ SANEX irradiation was a collaborative experiment between the United States Department of Energy-Nuclear Energy Material Recovery and Waste Form Development (MRWFD) and the European Union Safety of Actinide Separation Processes (SACSESS) ${ }^{14}$ programs. The results 
presented here indicate that the radiolytic performance of the TODGA/SO 3 -Ph-BTP, $i$-SANEX process under test loop radiolysis is much better than expected based on single-phase batch irradiation experiments.
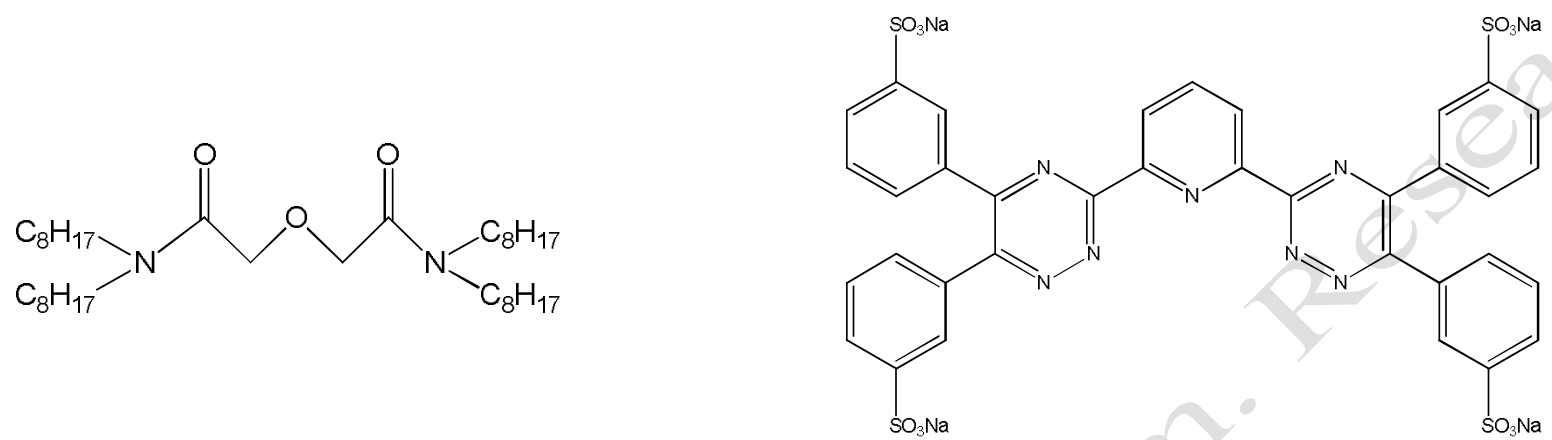

Figure 1. Structures of TODGA (left) and SO3-Ph-BTP (right).

\section{EXPERIMENTAL}

\section{Solvent System}

Two solvent systems were irradiated, based on prior work with $i$-SANEX formulation. ${ }^{6}$ The first system represented the group actinide/lanthanide extraction conditions using an organic phase consisting of $0.2 \mathrm{M}$ TODGA/5\% 1-octanol/dodecane in contact with an aqueous phase consisting of 4.5 $\mathrm{M} \mathrm{HNO}_{3}$. The octanol serves as a phase modifier which prevents the third phase formation that can occur during high metal loading of the organic phase. ${ }^{15}$ The second system represented stripping conditions, and consisted of the same $0.2 \mathrm{M}$ TODGA/5\% 1octanol/dodecane organic phase in contact with an aqueous phase consisting of $0.018 \mathrm{M} \mathrm{SO}_{3}-\mathrm{Ph}$ BTP/0.35 $\mathrm{M} \mathrm{HNO}_{3}$. The structures of both compounds are shown in Figure 1. For both solvent systems, approximately 10 ppm concentrations of stable $\mathrm{Sr}\left(\mathrm{NO}_{3}\right)_{2}, \mathrm{RuCl}_{3}$, and $\mathrm{Nd}\left(\mathrm{NO}_{3}\right)_{3}$ salts were added to the aqueous phase and were present during the irradiations, to evaluate the effect of these fission product elements on the process.

\section{Test Loop and Batch Irradiations}


The radiation source was an MDS Nordion GammaCell 220 Excel self-contained ${ }^{60}$ Co gamma irradiator. An array of ${ }^{60} \mathrm{Co}$ pins surrounds the irradiator sample chamber with a center-line $\gamma$-ray dose rate at the time of these experiments of $4.5 \mathrm{kGy} / \mathrm{hr}$, based on standard Fricke dosimetry. The irradiated volume is contained in a coil of borosilicate glass tubing ( $\sim 75$ 'length, 0.375 ' OD, 0.202 " ID) inside the sample chamber. Glass was chosen to minimize gamma heating of the tubing directly exposed to high $\gamma$-dose rates. The tubing outside the sample chamber is composed of 316 stainless steel. The connections between the stainless steel tubing and the glass coil were composed of Teflon ${ }^{\mathrm{TM}}$-lined Tygon ${ }^{\circledR}$ tubing during the experiment involving the simulated extraction stage, and of stainless steel convoluted tubing during the experiment simulating the strip stage. The significance of this change will be discussed in the results section of this report.

The absorbed dose to the experimental solution cannot be determined based merely on the center-line sample chamber dose rate since the dose rate varies about $\pm 20 \%$ across the volume of the sample chamber, and since a fraction of the total test loop solution resides outside the sample chamber in pumps and tubing at any given time during the experiment. Therefore, the effective dose rate experienced by the solution was measured prior to these experiments by recirculating a methyl red solution through the test loop and measuring its photo-bleaching at $430 \mathrm{~nm}$ by UV/Vis spectroscopy. ${ }^{16}$ The decrease in methyl red absorbance in the test loop was converted to an absorbed dose by reference to batch methyl red solutions that were irradiated at characterized positions in the sample chamber. The dose rates at these characterized positions were known based on standard Fricke dosimetry. The effective gamma dose rate to the test loop solutions was $3.3 \mathrm{kGy} / \mathrm{hr}$.

To best simulate a solvent extraction process, the test loop aqueous and organic phases were mixed using a centrifugal contactor (CINC V-02, USA) with the rotor replaced by a four vane 
mixing paddle. The organic and aqueous phases were metered into the mixing region of the contactor at an aqueous to organic phase volume ratio of $\mathrm{O} / \mathrm{A}=1$. The mixed phases were pumped through the test loop by a magnetic drive gear pump and flowed through the glass coil in the irradiator sample chamber and returned to the inlet of the centrifugal contactor where the phases were again mixed and circulated back through the loop. In-line tube mixers, shown as "static mixers" (TAH Industries, Inc.) were used to ensure that the dispersion did not disengage during recirculation. The flowrate of the dispersion was in the range of $1.5 \mathrm{~L} / \mathrm{min}$ to maintain turbulent flow, thus further ensuring that the phases remained mixed. A flow sight glass allowed verification of the extent of mixing in the test loop. The two phase dispersion continued to circulate for a time sufficient to deliver the desired absorbed dose, based on the effective dose rate determined above. A second stainless steel coil which is external to the irradiator sample chamber and held in a thermostated water bath was used maintain a constant temperature in the test loop. For these experiments that coil was used to maintain the test loop temperature at $25 \pm$ $0.5^{\circ} \mathrm{C}$. A schematic view of the test loop is shown in Figure 2 .

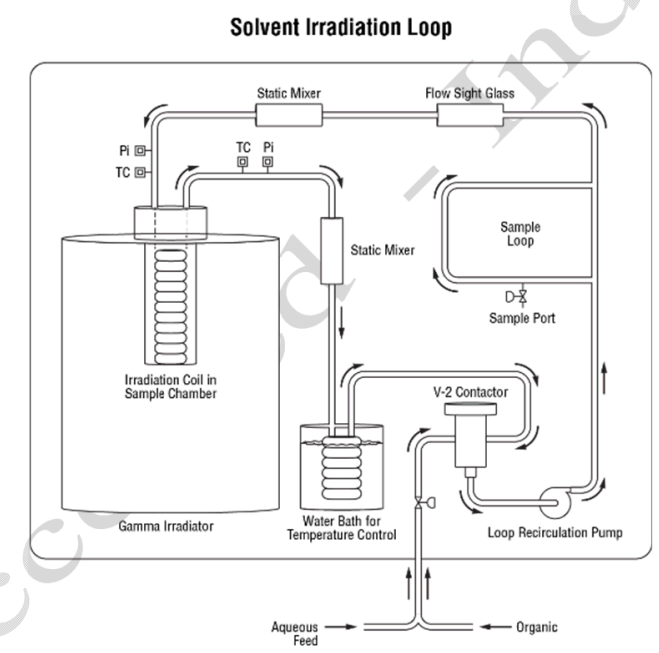

Figure 2. Schematic diagram of the radiolysis test loop. 
Prior to the test loop irradiations, scoping samples were irradiated in a batch, static configuration for comparison. These biphasic batch samples were held in septa-sealed scintillation vials and exposed to gamma irradiation at characterized positions in the irradiator sample chamber. Samples irradiated in sealed containers experience a de-oxygenated environment after exposure to only a small absorbed dose due to the scavenging of dissolved oxygen by radiolytically produced solvated electrons. ${ }^{17}$ However, in the test loop, and in an actual process, the systems are not sealed and air is replenished during mixing and recirculation. Therefore, the batch samples here were sparged with air during irradiation at a flow rate of 1 $\mathrm{cm}^{3} \cdot \mathrm{min}^{-1}$ using mass flow controllers (Sierra Instruments). This flow rate was selected to maintain the dissolved oxygen concentration without loss in sample volume due to evaporation.

\section{Solvent Extraction Performance}

In order to gauge the effect of gamma radiolysis upon the extraction performance of the solvents used in this study, the distribution ratios of americium $\left(D_{\mathrm{Am}}\right)$, cerium $\left(D_{\mathrm{Ce}}\right)$, and europium $\left(D_{\mathrm{Eu}}\right)$ were measured as a function of the absorbed dose in post-irradiation samples. Radiotracers $\left({ }^{241} \mathrm{Am},{ }^{139} \mathrm{Ce}\right.$, and $\left.{ }^{152,154} \mathrm{Eu}\right)$ were obtained from laboratory stocks. Samples of the irradiated aqueous and organic phases were obtained during test loop grab sampling campaigns and the radiotracers were added to the post-irradiation aqueous phases. Following the addition of the radiotracers, the phases were mixed for thirty minutes using a large capacity mixer (GlassCol) and centrifuged to separate. The activity of radiotracers present in the organic and aqueous phases was determined by gamma spectroscopy. An organic to aqueous phase volume ratio of $\mathrm{O} / \mathrm{A}=1$ was used for all contacts. The solvent extraction experiments were performed at ambient temperature $\left(21 \pm 2{ }^{\circ} \mathrm{C}\right)$. The distribution ratios were determined by calculating the ratio of activity in the organic and aqueous phases, $D=\mathrm{A}_{\mathrm{org}} / \mathrm{A}_{\mathrm{aq}}$. All distribution ratio determinations 
were performed in triplicate. The relative percent precision of distribution ratio values in the range of $D=0.01-100$ was less than $10 \%$. For distribution ratio values $D>500$, the relative percent precision was generally greater than $50 \%$.

In addition to the radiotracers, the distribution ratios $D_{\mathrm{Sr}}, D_{\mathrm{Ru}}$, and $D_{\mathrm{Nd}}$ for stable metals added prior to the irradiation of the aqueous phases were determined, as well as the concentrations of the selected stainless steel corrosion products $\mathrm{Cr}$, Mo, Ti and Ni by inductively coupled plasmamass spectrometry (ICP-MS). The aqueous phases were analyzed directly after a suitable dilution. The organic phases were digested using a microwave digestion apparatus and analyzed following dilution of the digested sample. The distribution ratios were determined by calculating the ratio of the concentrations in the organic and aqueous phases, $D=\mathrm{C}_{\mathrm{org}} / \mathrm{C}_{\mathrm{aq}}$. Spike recoveries for the ICP-MS analyses were $100 \pm 5 \%$ and the relative percent precisions of the ICP-MS determinations for all analytes was less than $3 \%$.

An additional important solvent extraction parameter that may be affected by radiolysis is the phase disengagement time of the mixed phase dispersion. Typically, this parameter is measured as the dispersion number (a dimensionless number used to characterize how quickly two immiscible phases separate after mixing). ${ }^{18}$ Values of the dispersion number were not determined over the entire course of the irradiation experiment due to sample volume constraints. Rather, at each grab sampling campaign, the time required for phase disengagement was measured by placing approximately $60 \mathrm{~mL}$ of the aqueous/organic dispersion in a graduated cylinder and noting the time required for phase separation. A dispersion number was measured only at the completion of each test loop irradiation.

Gas chromatography (GC) analyses were performed on a Thermo Scientific Trace ULTRA gas chromatograph with a flame ionization detector (FID). The chromatograms were processed using 
Thermo Scientific Xcalibur software. The chromatographic separations were carried out utilizing a Thermo Scientific TG-35MS capillary column ( $30 \mathrm{~m}$ x $0.32 \mathrm{~mm}$ ID x $0.5 \mu \mathrm{m}$ film). Analytical conditions were set at $2.0 \mathrm{~mL} / \mathrm{min}$ constant flow with helium as the carrier gas and an $80 \mathrm{~mL} / \mathrm{min}$ split flow. Oven operating conditions started with a 2 min hold at $70^{\circ} \mathrm{C}$, followed by a ramp at $20^{\circ} \mathrm{C} / \mathrm{min}$ to $240^{\circ} \mathrm{C}$ then $40^{\circ} \mathrm{C} / \mathrm{min}$ to $280^{\circ} \mathrm{C}$, finished with an $8.25 \mathrm{~min}$ hold at $280^{\circ} \mathrm{C}$. A Thermo AS3000 auto sampler was used for all injections, employing a $1 \mu \mathrm{L}$ hot injection with the inlet set at $250{ }^{\circ} \mathrm{C}$ and 5 second pre-injection dwell time. The FID was held constant at $250^{\circ} \mathrm{C}$. The fuel gas for the FID is a mixture of $350 \mathrm{~mL} / \mathrm{min}$ air and $35 \mathrm{~mL} / \mathrm{min}$ hydrogen with $30 \mathrm{~mL} / \mathrm{min}$ nitrogen as a makeup gas. Chromatographic grade hexane was used for the preparation of all standards and samples

Ion chromatography (IC) with conductivity detection was used to quantify sulfate anion concentration in the irradiated solvent system. The IC analyses were performed using a Dionex (Sunnyvale, CA, USA) ICS-5000 ion chromatograph equipped with an eluent generator, an autosampler, a quaternary gradient pump with degasser, conductivity detector, an anion selfregenerating suppressor (ASRS), a CR-ATC continuously regenerated anion trap column, and an ASRS 300 anion suppressor. All columns and other consumables were obtained from Dionex. An IonPac AS-11HC (4mm x 150mm) with AG-11HC guard column (4mm x 35mm) was used. The flow rate was isocratic at $1.0 \mathrm{~mL} / \mathrm{min}$. The step gradient was maintained using an EluGen II $\mathrm{KOH}$ cartridge and eluent generator. IC quality water was used for the preparation of all eluents, standards, and samples.

High Performance Liquid Chromatography (HPLC) was used to quantify TODGA concentration in the irradiated solvent systems. The HPLC analyses were performed using a Dionex (Sunnyvale, CA, USA) ICS-5000 ion chromatograph equipped with an autosampler, a 
quaternary pump with degasser, a photo-diode array detector, and Chromeleon 7 software. HPLC analyses were carried out using an eluent comprised of a 70:30 ratio of eluent A and eluent B. Eluent A consisted of $4 \%(\mathrm{v} / \mathrm{v})$ 1-octanol in 2-propanol. Eluent B consisted of $0.1 \%(\mathrm{v} / \mathrm{v})$ formic acid in water. The chromatographic separation was achieved with a $\mathrm{C}_{18}$ reverse-phase (RP-C 18 ) column (Supelco, $25 \mathrm{~cm} \times 4.6 \mathrm{~mm}, 5 \mu \mathrm{m}$ ) with a flow rate of $0.8 \mathrm{~mL} / \mathrm{min}$. The column temperature was maintained at $50{ }^{\circ} \mathrm{C}$. The absorbance of the column effluent was monitored at $220 \mathrm{~nm}$. HPLC quality water was used for the preparation of all eluents, standards, and samples.

\section{RESULTS AND DISCUSSION}

Batch Irradiation of $0.2 \mathrm{M} \mathrm{TODGA/5 \%}$ 1-octanol/dodecane/4.5 $\mathrm{M} \mathrm{HNO}_{3}$

The change in $D_{\mathrm{Am}}, D_{\mathrm{Ce}}$ and $D_{\mathrm{Eu}}$ as a function of absorbed dose up to a maximum of $650 \mathrm{kGy}$ for the batch irradiation of the TODGA solvent in contact with $4.5 \mathrm{M} \mathrm{HNO}_{3}$ is shown in Figure 3. The radiotracers were strongly extracted into the organic phase over the entire absorbed dose range, with efficiency similar to the un-irradiated solution. With such high distribution ratios, the uncertainty associated with the measurement is high, because there is very little activity in the post-contact aqueous phase to measure, and error bars are not shown in Figure 3. However, the data indicate that gamma irradiation of this TODGA solvent system under aerated static conditions in the presence of the aqueous phase did not result in any diminution in the efficiency of extraction for americium, cerium or europium.

Test Loop Irradiation of 0.2 M TODGA/5\% 1-octanol/dodecane/4.5 $\mathrm{M} \mathrm{HNO}_{3}$

The same TODGA solvent formulation in contact with the same 4.5 $\mathrm{M} \mathrm{HNO}_{3}$ was irradiated in the test loop to a maximum absorbed dose of $878 \mathrm{kGy}$. The effect on $D_{\mathrm{Am}}, D_{\mathrm{Ce}}$ and $D_{\mathrm{Eu}}$ was similar to the batch irradiations, even at the higher absorbed dose. No measurable variation in the 
measured distribution ratios as a function of absorbed dose occurred. These results are also shown in Figure 3.

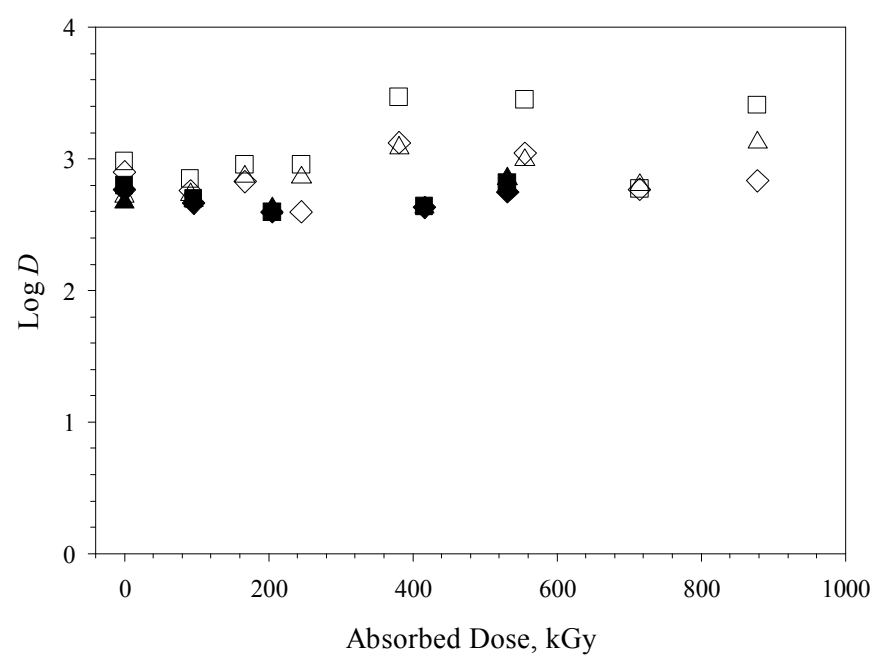

Figure 3. Plot of log distribution ratios versus absorbed dose. 0.2 M TODGA / $5 \%$ 1-octanol in dodecane in contact with 4.5 $\mathrm{M} \mathrm{HNO}_{3}$. Test loop irradiation: Am (open triangles); Eu (open squares); Ce (open diamonds). Static irradiation: Am (filled triangles); Eu (filled squares); Ce (filled diamonds).

The fission products $\mathrm{Nd}, \mathrm{Ru}$ and $\mathrm{Sr}$ were added as stable metals to the aqueous solution prior to the test loop irradiation. Their distribution ratios were also measured as a function of absorbed dose and are shown in Figure 4. The aqueous phase neodymium concentrations for all samples were below ICP-MS detection limits; therefore, the calculated values of $D_{\mathrm{Nd}}$ in Figure 4 actually represent a lower limit. It can be seen that neodymium was quantitatively extracted even at the maximum absorbed dose. This result is consistent with those reported above for the static irradiations. These findings are, also, consistent with those of Modolo et al. ${ }^{10}$ In that work, solutions of 0.2 M TODGA in the aliphatic diluent TPH were $\gamma$-irradiated at a dose rate of 1.9 $\mathrm{kGy} / \mathrm{hr}$ with or without contact with $3 \mathrm{M} \mathrm{HNO}_{3}$. They also reported that $D_{\mathrm{Am}}$ and $D_{\mathrm{Eu}}$ were little 
affected at an absorbed dose of $600 \mathrm{kGy}$, although $D_{\mathrm{Am}}$ appeared to decline slightly at absorbed doses as high as $1000 \mathrm{kGy}$. In that work, 1-octanol was not present in the solvent formulation; instead, $0.5 \mathrm{M}$ tributylphosphate (TBP) was used as a modifier.

In-depth studies of the radiation chemistry of the diglycolamides TODGA, tetraethyhexyldiglycolamide (TEHDGA), and the methyl-substituted derivatives MeTODGA and $\mathrm{Me}_{2}$ TODGA were recently published for batch irradiated samples of $0.05 \mathrm{M}$ diglycolamide (DGA)/dodecane solutions. ${ }^{11,11 \mathrm{e}}$ These solutions did not contain phase modifiers. In those reports, similar pseudo-first-order rate constants (dose constants, $d$ ) for the loss of ligand concentration with increasing absorbed dose were measured, for DGAs with and without contact to the acidic aqueous phase and with and without air sparging. This is consistent with Modolo et al. ${ }^{10}$ who found a lack of effect of the acidic aqueous phase during irradiation based on invariant $D_{\mathrm{Am}}$ and $D_{\mathrm{Eu}}$ values for samples with and without contact to an acidic aqueous phase. However, the dose constants reported by Galan et al. ${ }^{11 \mathrm{f}}$ and Zarzana et al. ${ }^{11 \mathrm{e}}$ predict that most of the TODGA would be destroyed at absorbed doses as high as those used by Modolo et al. ${ }^{10}$ and in this work. Therefore, the metal distribution ratios would be expected to decline. For example, assuming a dose constant of $4 \times 10^{-3} \mathrm{kGy}^{-1}$ as reported by Zarzana et al. ${ }^{11 \mathrm{e}}$ for TODGA, the first order rate equation may be used to calculate the remaining ligand concentration:

$$
[\text { TODGA }]=[\text { TODGA }]_{\mathrm{o}} \mathrm{e}^{-(d \mathrm{D})}
$$

where $\mathrm{D}$ is the absorbed dose in kGy. Starting with $0.2 \mathrm{M}$ TODGA, the final ligand concentration at an absorbed dose of $878 \mathrm{kGy}$ is only $0.008 \mathrm{M}$. While it is tempting to suggest that the extraction efficiency remains invariant with absorbed dose because octanol (or TBP in Modolo et al. ${ }^{10}$ ) protects TODGA from radiolytic degradation, TODGA radiolysis products may complex and extract the metals to maintain extraction efficiency. In fact, a product DGA created 
by the radiolytic dealkylation of TODGA has been reported by Galán, ${ }^{11 \mathrm{f}}$ Galán, ${ }^{1 \mathrm{~d}}$ and Zarzana. ${ }^{11 \mathrm{e}}$

For the non-f-element fission products, $D_{\mathrm{Ru}}$ increased slightly (Figure 4) with increasing absorbed dose during the test loop TODGA irradiation, which may indicate either that a radiolysis product capable of extracting ruthenium was produced during the irradiation experiment, or, that ruthenium redox chemistry is occurring in irradiated solution. Previously, it has been shown that increased $D_{\mathrm{Ru}}$ values occurred for solvent extraction under oxidizing conditions. ${ }^{19}$ However, the increase here in $D_{\mathrm{Ru}}$ was minor, and the value remained $<1$ even at the highest absorbed dose investigated.

A decrease of $D_{\mathrm{Sr}}$ over the course of the irradiation was found, and is also shown in Figure 4. This unexpected result may be attributable to the build-up of fluoride ion in the aqueous phase during this test loop run. For this run only, a short Telfon ${ }^{\circledR}$-lined Tygon ${ }^{\circledR}$ tubing joint was used to connect the glass irradiation coil to the stainless steel tubing in the balance of the loop. Unfortunately, while only a small amount of the tubing extended into the upper portion of the irradiation chamber, it underwent significant radiolytic degradation over the course of the TODGA $/ \mathrm{HNO}_{3}$ irradiation. Ion chromatographic analysis of the irradiated nitric acid phase (see Figure S1 supporting information) demonstrated that fluoride ion was introduced into the aqueous phase as a function of increasing gamma dose, reaching a final concentration of about 4 ppm $(0.2 \mathrm{mM})$. Following the completion of this experiment, the Tygon ${ }^{\circledR}$ tubing was replaced with stainless steel convoluted tubing for the subsequent TODGA/SO $3-\mathrm{Ph}-\mathrm{BTP}$ experiment. As will be discussed later, this eliminated the fluoride ion contamination, and this anomalous $D_{\mathrm{Sr}}$ behavior was not again seen. A similar effect due to fluoride complexation of neodymium would be reasonable to expect. However, the very high values of neodymium distribution ratios indicate 
that the TODGA extractant is able to effectively compete for the neodymium present in aqueous phase.

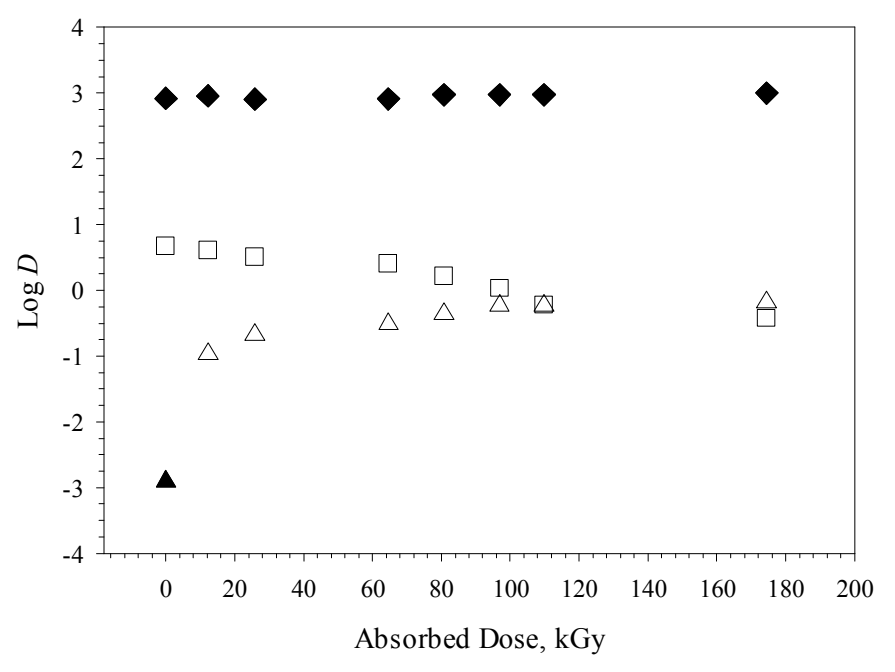

Figure 4. Plot of log distribution ratio values of $\mathrm{Sr}$ (squares), $\mathrm{Ru}$ (triangles), and $\mathrm{Nd}$ (diamonds) as a function of absorbed dose for the test loop irradiation of the TODGA $/ \mathrm{HNO}_{3}$ system. The value of DNd (solid diamonds) represents a lower limit due to aqueous neodymium concentrations below the ICP-MS detection limit. The initial, unirradiated, $\mathrm{D}_{\mathrm{Ru}}$ (solid triangle) is calculated based on the concentration of ruthenium extracted being below detection limit.

The aqueous and organic phases from the TODGA $/ \mathrm{HNO}_{3}$ test loop irradiation were also analyzed by ICP-MS for chromium, nickel, titanium and molybdenum; selected corrosion products of stainless steel (see Figure S2 supporting information). The aqueous concentrations of chromium and nickel increased with increasing dose, although they were apparently not extracted into the organic phase. Trace amounts of titanium that exhibited no trend with absorbed dose were found in both phases. The final aqueous concentrations of chromium and nickel at the highest absorbed dose were 0.4 and $0.5 \mathrm{mM}$, respectively. In contrast, molybdenum was not 
detected in the aqueous phase. However, examination of the concentration of molybdenum present in the organic phase showed that it was quantitatively extracted by the TODGA solvent, with a final organic phase concentration of $0.1 \mathrm{mM}$. Although the concentration of these corrosion products of stainless steel are discussed with respect to absorbed dose, it is not suggested that the effect is radiolytic. Rather, this may merely be a function of the duration of exposure of system components to the nitric acid solution. These data were collected mainly for the purposes of comparison to the TODGA/SO 3 - $\mathrm{Ph}-\mathrm{BTP}$ system, where it was hypothesized that radiolysis of the sulfonated BTP might generate sulfuric acid. This is discussed in more detail later.

Finally, variation in the time required for phase disengagement as a function of absorbed dose for the TODGA $/ \mathrm{HNO}_{3}$ test loop irradiation samples was examined. Disengagement time increased from approximately $80 \mathrm{sec}$ at zero absorbed dose to $\sim 140 \mathrm{sec}$ at $200 \mathrm{kGy}$, and then exhibited a subsequent gradual decrease as the irradiation continued; with a final value of about $120 \mathrm{sec}$ at the highest absorbed dose of $878 \mathrm{kGy}$. The dispersion number at the completion of the test was $N_{\mathrm{Di}}=9 \times 10^{-4}$. Values between $(8-16) \times 10^{-4}$ are considered acceptable in typical stagewise solvent extraction equipment, and therefore it is concluded irradiation of the TODGA $/ \mathrm{HNO}_{3}$ solvent system to an absorbed dose of $878 \mathrm{kGy}$ did not adversely affect its hydrodynamic performance. ${ }^{18}$

Test Loop Irradiation of 0.2 M TODGA/5\% 1-octanol/dodecane/0.018 M SO3-Ph-BTP/0.35 M $\mathrm{HNO}_{3}$

The second test loop irradiation utilized the same organic phase composition, but the aqueous phase was replaced with a solution containing $0.018 \mathrm{M} \mathrm{SO}_{3}$-Ph-BTP dissolved in $0.35 \mathrm{M} \mathrm{HNO}_{3}$. The aqueous-soluble BTP acts as an actinide hold-back reagent, and its addition affords the 
desired An(III)/Ln(III) separation. Based upon the results of Galan et al., ${ }^{12}$ a lower total absorbed dose of $175 \mathrm{kGy}$ was delivered to the BTP-containing solvent system. The same suite of analyses was performed as for the TODGA/ $\mathrm{HNO}_{3}$ system. Over the course of the irradiation, the aqueous BTP-phase darkened considerably. The color changed from wine-red in unirradiated samples to opaque black. While no sulfurous odors were noted during the course of the irradiation, the significant darkening of the aqueous phase was attributed to the radiolytic degradation of the $\mathrm{SO}_{3}$-Ph-BTP hold-back reagent.

The values of $D_{\mathrm{Am}}, D_{\mathrm{Eu}}$, and $D_{\mathrm{Ce}}$ determined using samples taken from the TODGA/SO 3 - $\mathrm{Ph}$ BTP test loop irradiation are presented in Figure 5. As expected all the distribution ratios are lower in magnitude than in the absence of the aqueous hold-back reagent, with $D_{\mathrm{Am}}$ especially suppressed to values $<1$. It can also be seen that the americium and cerium and europium distribution ratios for the irradiated TODGA/SO 3 - Ph-BTP solvent system do not exhibit a dependence upon absorbed dose that is consistent with significant radiolytic degradation of the $\mathrm{SO}_{3}-\mathrm{Ph}$-BTP ligand. Thus, in spite of the dramatic darkening of the irradiated aqueous phase, the separation factors between the actinide and these two lanthanides were essentially unchanged over this absorbed dose range.

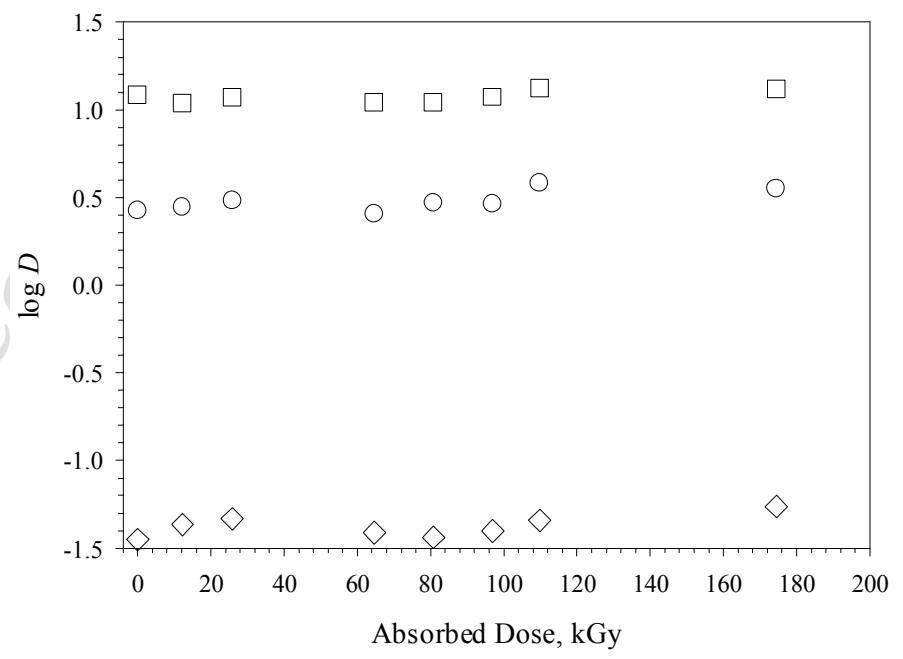


Figure 5. Plot of log distribution ratio values of Am (diamonds), Eu (squares), and Ce (circles) determined as a function of absorbed dose for the test loop irradiation of the TODGA $/ \mathrm{SO}_{3}-\mathrm{Ph}$ BTP solvent system.

The $D_{\mathrm{Nd}}, D_{\mathrm{Ru}}$ and $D_{\mathrm{Sr}}$ for the added stable fission product metals were also determined for this system and are shown in Figure 6 . As expected, $D_{\mathrm{Nd}}$ is lower than in the absence of the $\mathrm{SO}_{3}-\mathrm{Ph}$ BTP, and similarly to americium, cerium and europium, it is invariant with absorbed dose. In contrast to the results reported above for the TODGA/ $\mathrm{HNO}_{3}$ system, ruthenium was not extracted in the presence of the $\mathrm{SO}_{3}-\mathrm{Ph}-\mathrm{BTP}$, and the $D_{\mathrm{Sr}}$ did not vary over the course of the irradiation. The strontium results are attributed to the absence of fluoride in the system following replacement of the plastic tubing connections to the irradiation coil with stainless steel.

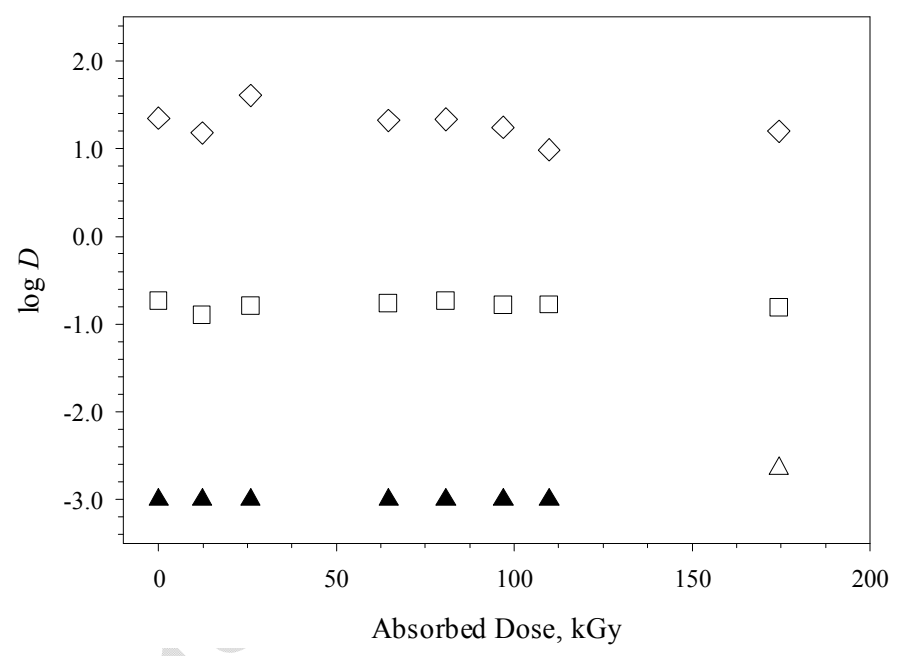

Figure 6. Plot of distribution ratio values of $\mathrm{Sr}$ (squares), $\mathrm{Ru}$ (triangles), and $\mathrm{Nd}$ (diamonds) determined by ICP-MS as a function of absorbed dose for the test loop irradiation of the TODGA/SO $\mathrm{S}_{3}-\mathrm{Ph}-\mathrm{BTP}$ solvent system. The values of $D_{\mathrm{Ru}}$ (solid triangles) represents an upper limit due to organic ruthenium concentrations below the ICP-MS detection limit. 
The aqueous and organic phases from the TODGA $/ \mathrm{SO}_{3}-\mathrm{Ph}-\mathrm{BTP}$ test loop irradiation were analyzed by ICP-MS for chromium, nickel, titanium and molybdenum; selected corrosion products of stainless steel (see Figure S3 Supporting Information). For the TODGA/nitric acid system at $\sim 100 \mathrm{kGy}$ absorbed dose, the concentrations of chromium, nickel and titanium are approximately 4, 5, and $<1 \mathrm{ppm}$, respectively. At the same absorbed dose, the concentration of chromium, nickel and titanium are approximately 9,8 , and $1 \mathrm{ppm}$, respectively, in the presence of the $\mathrm{SO}_{3}-\mathrm{Ph}-\mathrm{BTP}$. The molybdenum concentration measured in the organic phase at $\sim 100 \mathrm{kGy}$ absorbed dose was approximately the same for both systems. In aggregate these results may indicate a greater rate of corrosion in the presence of the $\mathrm{SO}_{3}-\mathrm{Ph}-\mathrm{BTP}$, suggesting that sulfuric acid is produced by radiolytic desulfonation of the hold-back reagent. However, the effect was not dramatic. The degradation of the $\mathrm{SO}_{3}-\mathrm{Ph}-\mathrm{BTP}$ is not so severe as to adversely impact the targeted actinide/lanthanide separation, at least over this absorbed dose range. These results are more optimistic for the development of a process than those reported by Galan et al. ${ }^{12}$ In that work, $D_{\mathrm{Am}}$ climbed very quickly with absorbed dose, while $D_{\mathrm{Eu}}$ remained unchanged, resulting in a decrease in the desired separation factor, however, only the aqueous phase was irradiated in that study.

In order to develop a better understanding of radiolytic degradation of this $i$-SANEX solvent system, the variation in the solvent composition as a function of absorbed dose was determined for both test loop irradiation experiments. The concentration of TODGA in the organic samples was determined by HPLC and concentration of 1-octanol present in the organic samples was determined by gas chromatography. The variation in the TODGA concentration with absorbed dose is presented in Figure 7 and the variation in 1-octanol concentration with absorbed dose is presented in Figure 8. For both the TODGA $/ \mathrm{HNO}_{3}$ and TODGA/SO $\mathrm{S}_{3}-\mathrm{Ph}-\mathrm{BTP}$ irradiation 
experiments, the same dependence of TODGA or octanol concentration on absorbed dose was observed. These data indicate that both TODGA and octanol are subject to radiolytic degradation. The concentration of 1-octanol in the un-irradiated solvent $(\sim 0.25 \mathrm{M})$ is lower than the targeted nominal concentration of $0.32 \mathrm{M}$ or $5 \%(\mathrm{v} / \mathrm{v})$. This discrepancy is attributed to an error during the preparation of the solvent used for these studies. The results found here should not be significantly different than for the $5 \%$ octanol process solution.

Interestingly, the rate of TODGA degradation in these experiments is only approximately $25 \%$ the rate reported by Zarzana et al. ${ }^{11 \mathrm{e}}$ for the radiolytic degradation of TODGA dissolved in dodecane in which no octanol was present. While it is tempting to conclude that the octanol present in the solvent used in this work is acting to inhibit the radiolytic degradation of TODGA, the preliminary nature of the data makes such an assertion difficult to support. It should be noted that Galan et al., ${ }^{11 \mathrm{~d}}$ reported that irradiation of an alkane solvent containing $5 \%$ octanol showed a less adverse effect on $D_{\mathrm{Am}}$ and $D_{\mathrm{Eu}}$ as compared to an untreated octanol-containing solvent. This radio-protection effect of $\mathrm{HNO}_{3}$ did not occur for an octanol-free alkane organic phase, and may be related to the extraction of $\mathrm{HNO}_{3}$ in the presence of the alcohol. ${ }^{20}$

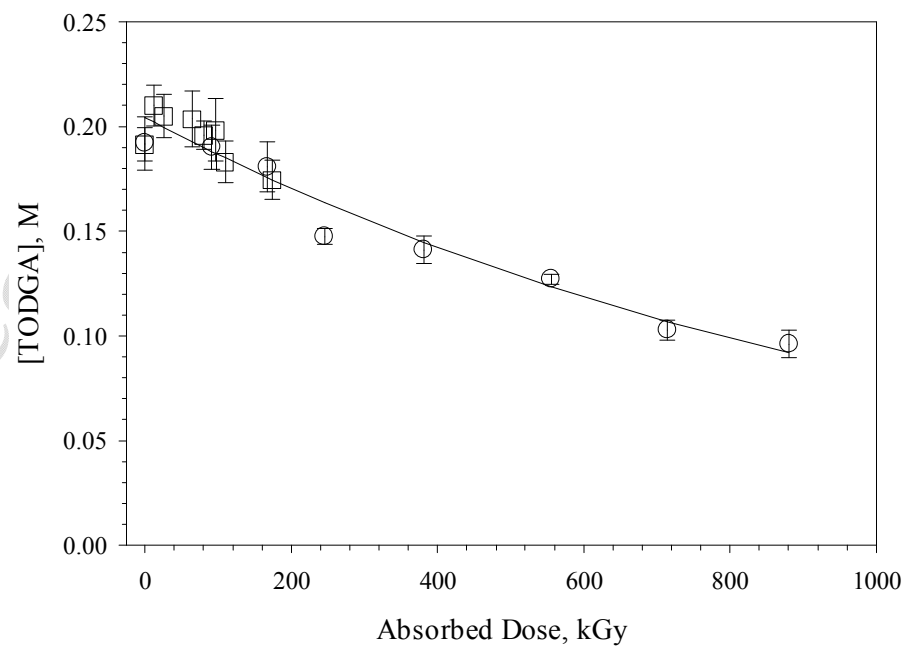


Figure 7. Plot of the concentration of TODGA versus absorbed dose for the test loop irradiation of the TODGA/nitric acid (circles) and TODGA/SO3-Ph-BTP + nitric acid (squares) solvent systems. The solid line is an exponential fit to both data sets. Equation of best-fit line: $(2.04 \pm$ $0.03) \times 10^{-1} \exp -(9.01 \pm 0.572) \times 10^{-4} \times, R^{2}=0.980$.

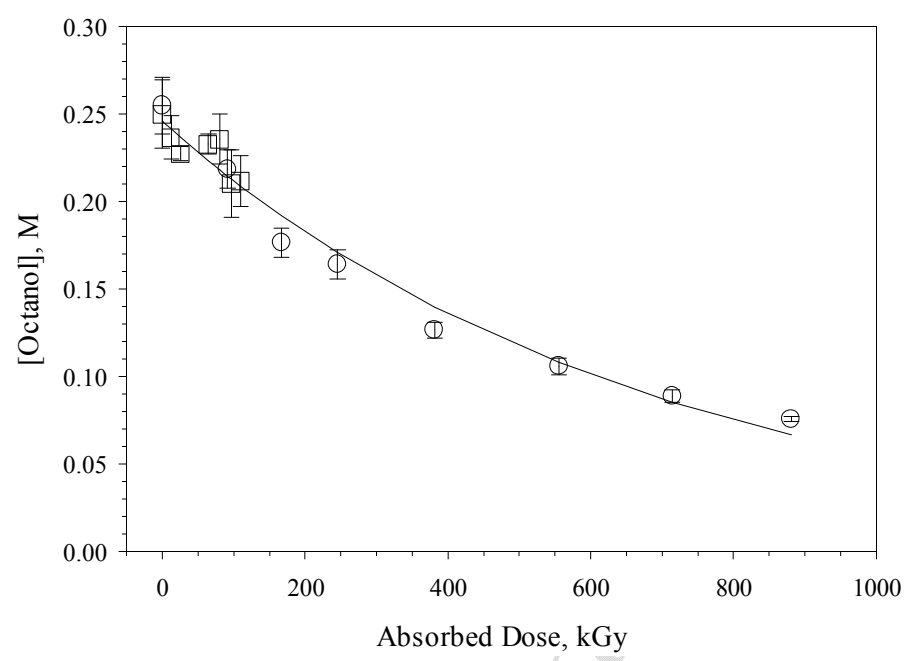

Figure 8. Concentration of 1-octanol versus absorbed dose for the test loop irradiation of the $i$ SANEX solvent in contact with $4.5 \mathrm{M} \mathrm{HNO}_{3}$ (circles) and the i-SANEX solvent in contact with $0.018 \mathrm{M} \mathrm{SO}_{3}$ - $\mathrm{Ph}-\mathrm{BTP} / 0.35 \mathrm{M} \mathrm{HNO}_{3}$ (squares). The solid line is an exponential fit to both data sets. Equation of best-fit line: $(2.46 \pm 0.042) \times 10^{-1} \exp -(1.48 \pm 0.070) \times 10^{-3} \times, R^{2}=0.980$.

Ion chromatographic (IC) analysis was used to determine the concentration of sulfate anion present in the $0.018 \mathrm{M} \mathrm{SO}_{3}-\mathrm{Ph}-\mathrm{BTP} / 0.35 \mathrm{M} \mathrm{HNO}_{3}$ samples as a function of absorbed dose. The IC results are presented in Figure 9. At zero absorbed dose, approximately $0.004 \mathrm{M}$ sulfate is present in the aqueous phase. As TODGA/0.018 $\mathrm{M} \mathrm{SO}_{3}-\mathrm{Ph}-\mathrm{BTP} / 0.35 \mathrm{M} \mathrm{HNO}_{3}$ solvent system is irradiated, the sulfate concentration increases to $0.007 \mathrm{M}$ at $170 \mathrm{kGy}$ absorbed dose. The increase 
in sulfate anion concentration and the color change occurring in the aqueous phase with increasing absorbed dose clearly indicates that radiolytic degradation of the $\mathrm{SO}_{3}-\mathrm{Ph}-\mathrm{BTP}$ holdback reagent occurs although the rate of degradation was not sufficient to impair performance of the solvent at these absorbed doses.

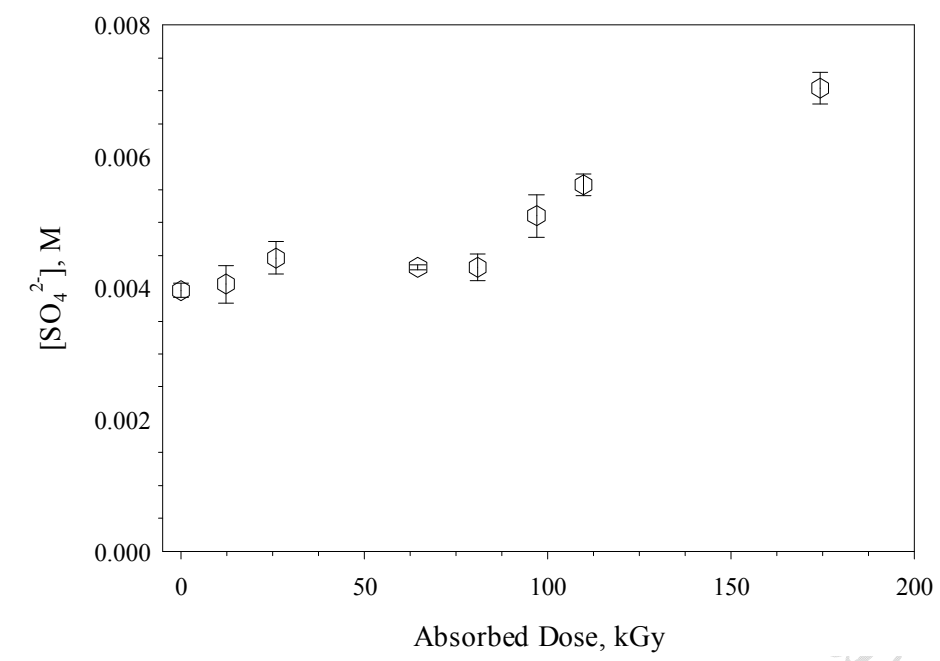

Figure 9. Concentration of sulfate anion (circles) versus absorbed dose the test loop irradiation of the $i$-SANEX solvent in contact with $0.018 \mathrm{M} \mathrm{SO}_{3}$-Ph-BTP/0.35 $\mathrm{M} \mathrm{HNO}_{3}$.

Finally, variation in the time required for phase disengagement as a function of absorbed dose for the TODGA $/ \mathrm{SO}_{3}-\mathrm{Ph}-\mathrm{BTP}$ test loop irradiation samples was measured. Disengagement time increased immediately upon irradiation from $35 \mathrm{sec}$ to nearly $90 \mathrm{sec}$, but then began a gradual trend to shorter separation times with absorbed dose; with a final value similar to that of the unirradiated material. The dispersion number at the conclusion of the irradiation was $\mathrm{N}_{\mathrm{Di}}=2 \times 10^{-3}$, indicating that excellent hydrodynamic behavior in typical stage-wise solvent extraction equipment is expected. ${ }^{18}$ 


\section{CONCLUSION}

The $i$-SANEX solvent formulation consisting of nominally $0.2 \mathrm{M}$ TODGA $/ 5 \%$ 1octanol/dodecane was $\gamma$-irradiated under realistic process conditions in the INL radiolysis test loop, in contact with both extraction and stripping aqueous phases. The extraction aqueous phase was 4.5 $\mathrm{M} \mathrm{HNO}_{3}$, and the stripping aqueous phase was $0.018 \mathrm{M} \mathrm{SO}_{3}-\mathrm{Ph}-\mathrm{BTP} / 0.35 \mathrm{M} \mathrm{HNO}$. When irradiated in contact with only the $4.5 \mathrm{M} \mathrm{HNO}_{3}$ phase, the TODGA solvent maintained excellent extraction performance for americium, cerium, europium and neodymium to a maximum absorbed dose of nearly 0.9 MGy. The results for preliminary static, batch irradiations were consistent with test loop findings. When the aqueous phase was changed to that containing the aqueous soluble BTP, the irradiated aqueous phase showed a dramatic color change, but this does not appear to have had adverse effects on solvent extraction performance. The distribution ratios for both the lanthanides and actinide were invariant with absorbed dose, and the separation factors were essentially unchanged to a maximum absorbed dose of $174 \mathrm{kGy}$. The results of ICPMS analysis of the aqueous and organic phases showed that ruthenium and strontium were not extracted in the presence of the irradiated BTP-aqueous phase. Molybdenum was extracted under both conditions. Further, the build-up of corrosion products of stainless steel in the presence of the BTP was not dramatically greater than in the nitric acid-only system. Phase separation times were not adversely affected by irradiation for either system. The results presented here indicate that the performance of the TODGA $/ \mathrm{SO}_{3}-\mathrm{Ph}-\mathrm{BTP}, i$-SANEX process under test loop radiolysis is much better than expected based on a literature report using single-phase batch irradiation experiments, and better than might be predicted based on DGA radiolysis studies alone.

These results very clearly indicate that an assessment of the effects of irradiation on solvent extraction systems depends upon measurements conducted under as realistic a scenario as 
possible. While the irradiation of simpler solutions containing individual solvent phases or components remains pertinent to a more fundamental understanding of their radiation chemistry, only studies of all the components in mixed dispersion such as that provided by the test loop described here can provide a realistic assessment of process system performance.

Areas for further research suggested by this work include a more fundamental investigation of the behavior of octanol in irradiated systems. Octanol may serve as a radical scavenger to protect TODGA during radiolysis, or may extract $\mathrm{HNO}_{3}$ into the organic phase which may then act as a radio-protection agent. Products analysis of the irradiated solvents will help to elucidate the radiolysis mechanisms in this multi-component system.

\section{Associated Content}

\section{Supporting Information.}

"This material is available free of charge via the Internet at http://pubs.acs.org."

Plot of fluoride concentration versus absorbed dose, plots of corrosion product concentration versus absorbed dose

\section{Author Information}

\section{Corresponding Author}

*E-mail: dean.peterman@inl.gov

\section{Notes}

The authors declare no competing financial interest.

\section{ACKNOWLEDGMENT}


This research was sponsored by the U.S. Department of Energy, Office of Nuclear Energy, through the Fuel Cycle Research and Development Program. Idaho National Laboratory is operated by Battelle Energy Alliance under DOE Idaho Operations Office contract DE-AC07$05 I D 14517$.

Insert Table of Contents Graphic and Synopsis Here

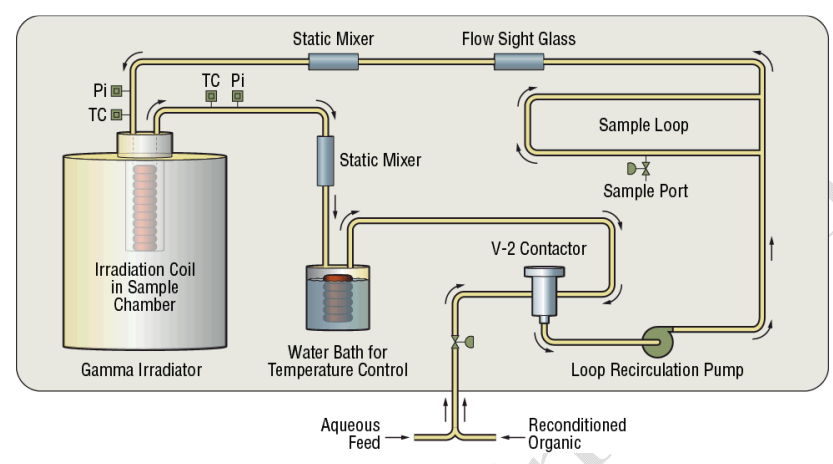




\section{REFERENCES}

1. Modolo, G.; Wilden, A.; Geist, A.; Magnusson, D.; Malmbeck, R., A review of the demonstration of innovative solvent extraction processes for the recovery of trivalent minor actinides from PUREX raffinate. Radiochimica Acta 2012, 100, 715.

2. Modolo, G.; Geist, A.; Miguirditchian, J. M. M., Minor actinide separations in the reprocessing of spent nucler fuels: recent advances in Europe. In Reprocessing and Recycling of Spent Nuclear Fuel, Taylor, R., Ed. Woodhead Publishing: Cambridge, UK, 2015.

3. Nilsson, M.; Nash, K. L., Review Article: A Review of the Development and Operational Characteristics of the TALSPEAK Process. Solvent Extraction and Ion Exchange 2007, 25, 665 701.

4. Gannaz, B.; Chiarizia, R.; Antonio, M. R.; Hill, C.; Cote, G., Extraction of Lanthanides(III) and Am(III) by Mixtures of Malonamide and Dialkylphosphoric Acid. Solvent Extraction and Ion Exchange 2007, 25, 313-337.

5. Gelis, A. V.; Lumetta, G. J., Actinide Lanthanide Separation Process-ALSEP. Industrial \& Engineering Chemistry Research 2014, 53, 1624-1631.

6. Wilden, A.; Modolo, G.; Kaufholz, P.; Sadowski, F.; Lange, S.; Sypula, M.; Magnusson, D.; Müllich, U.; Geist, A.; Bosbach, D., Laboratory-Scale Counter-Current Centrifugal Contactor Demonstration of an Innovative-SANEX Process Using a Water Soluble BTP. Solvent Extraction and Ion Exchange 2015, 33, 91-108.

7. (a) Panak, P. J.; Geist, A., Complexation and Extraction of Trivalent Actinides and Lanthanides by Triazinylpyridine N-Donor Ligands. Chemical Reviews 2013, 113, 1199-1236; 
(b) Aneheim, E.; Ekberg, C.; Fermvik, A.; Foreman, M.; Retegan, T.; Skarnemark, G., A TBP / BTBP - based GANEX Separation Process . Part 1 : Feasibility. Solvent Extraction and Ion Exchange 2010, 28, 437-458.

8. Geist, A.; Müllich, U.; Magnusson, D.; Kaden, P.; Modolo, G.; Wilden, A.; Zevaco, T., Actinide(III)/Lanthanide(III) Separation Via Selective Aqueous Complexation of Actinides(III) using a Hydrophilic 2,6-Bis(1,2,4-Triazin-3-Y1)-Pyridine in Nitric Acid. Solvent Extraction and Ion Exchange 2012, 30, 433-444.

9. Sasaki, Y.; Tsubata, Y.; Kitatsuji, Y.; Sugo, Y.; Shirasu, N.; Morita, Y.; Kimura, T., Extraction Behavior of Metal Ions by TODGA, DOODA, MIDOA, and NTAamide Extractants from $\mathrm{HNO}_{3}$ to n-Dodecane. Solvent Extraction and Ion Exchange 2013, 31, 401-415.

10. Modolo, G.; Asp, H.; Schreinemachers, C.; Vijgen, H., Development of a TODGA based Process for Partitioning of Actinides from a PUREX Raffinate Part I: Batch Extraction Optimization Studies and Stability Tests. Solvent Extraction and Ion Exchange 2007, 25, 703 721.

11. (a) Sugo, Y.; Sasaki, Y.; Tachimori, S., Studies on hydrolysis and radiolysis of N,N,N,Ntetraoctyl-3-oxapentane-1,5-diamide. Radiochimica Acta 2002, 90, 161-165; (b) Sugo, Y.; Izumi, Y.; Yoshida, Y.; Nishijima, S.; Sasaki, Y.; Kimura, T.; Sekine, T.; Kudo, H., Influence of diluent on radiolysis of amides in organic solution. Radiation Physics and Chemistry 2007, 76, 794-800; (c) Gujar, R. B.; Ansari, S. A.; Bhattacharyya, A.; Kanekar, A. S.; Pathak, P. N.; Mohapatra, P. K.; Manchanda, V. K., Radiolytic Stability of N,N,N',N'-Tetraoctyl Diglycolamide (TODGA) in the Presence of Phase Modifiers Dissolved in n-Dodecane. Solvent Extraction and Ion Exchange 2012, 30, 278-290; (d) Galán, H.; Núñez, A.; Espartero, A. G.; Sedano, R.; Durana, A.; de 
Mendoza, J., Radiolytic Stability of TODGA: Characterization of Degraded Samples under Different Experimental Conditions. Procedia Chemistry 2012, 7, 195-201; (e) Zarzana, C. A.; Groenewold, G. S.; Mincher, B. J.; Mezyk, S. P.; Wilden, A.; Schmidt, H.; Modolo, G.; Wishart, J. F.; Cook, A. R., A Comparison of the $\gamma$-Radiolysis of TODGA and T(EH)DGA Using UHPLC-ESI-MS Analysis. Solvent Extraction and Ion Exchange 2015, 33, 431-447; (f) Galan, H.; Zarzana, C. A.; Wilden, A.; Nunez, A.; Schmidt, H.; Egberink, R. J. M.; Leoncini, A.; Cobos, J.; Verboom, W.; Modolo, G.; Groenewold, G. S.; Mincher, B. J., Gamma-radiolytic stability of new methylated TODGA derivatives for minor actinide recycling. Dalton Transactions 2015, 44, 18049-18056.

12. Galán, H.; Munzel, D.; Núñez, A.; Müllich, U.; Cobos, J.; Geist, A. In Stability and Recyclability of $\mathrm{SO}_{3}$-Ph-BTP for i-SANEX Process Development, ISEC2014 International Solvent Extraction Conference, September 7 - 11, 2014; Würzburg, Germany, 2014.

13. Mincher, B. J.; Modolo, G.; Mezyk, S. P., The Effects of Radiation Chemistry on Solvent Extraction: 1. Conditions in Acidic Solution and a Review of TBP Radiolysis. Solvent Extraction and Ion Exchange 2009, 27, 1 - 25.

14. (a) Bourg, S.; Geist, A.; Narbut, J., SACSESS - the EUROATOM FP7 project on actinide separation from spent nuclear fuels. Nukleonika 2015, 60, 809 - 814; (b) Geist, A.; R., T.; Ekberg, C.; Guilbaud, P.; Modolo, G.; Bourg, S., The SACSESS hydrometallurgy domain - an overview. Procedia Chemistry 2016 accepted.

15. Geist, A.; Modolo, G. In TODGA Process Development: An Improved Solvent Formulation, Global 2009, Paris, France, September, 6-11, 2009; Paris, France, 2009; pp 10221026. 
16. Ajji, Z., Usability of aqueous solutions of methyl red as high-dose dosimeter for gamma radiation. Radiation Measurements 2006, 41, 438 - 442.

17. Mincher, B. J.; Mezyk, S. P., Radiation chemcial effects on radiochemistry: A review of examples important to nuclear power. Radiochimica Acta 2009, 97, 519 - 534.

18. Leonard, R. A., Solvent Characterization Using the Dispersion Number. Separation Science and Technology 1995, 30, 1103 - 1122.

19. Mincher, B. J.; Martin, L. R.; Schmitt, N. C., Diamylamylphosphonate Solvent Extraction of Am(VI) from Nuclear Fuel Raffinate Simulant Solution. Solvent Extraction and Ion Exchange 2012, 30, 445-456.

20. Geist, A., Extraction of Nitric Acid into Alcohol : Kerosene Mixtures. Solvent Extraction and Ion Exchange 2010, 28, 596-607. 

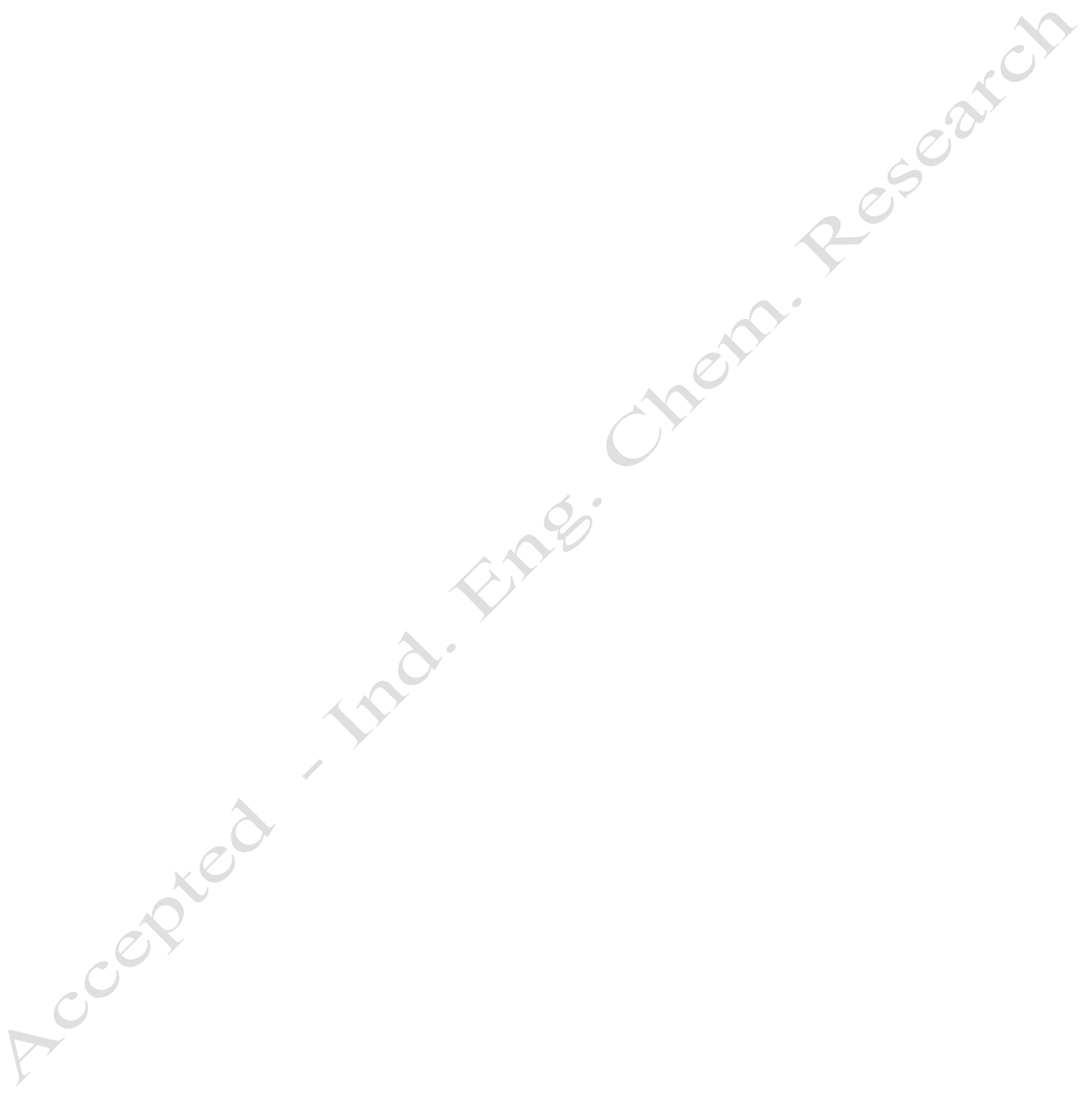